\title{
ZERO SETS OF FUNCTIONS FROM NON-QUASI-ANALYTIC CLASSES
}

\author{
R. B. DARST
}

\begin{abstract}
Any closed subset $E$ of the real numbers $R$ is the zero set of some $C^{\infty}$-function $f$. One can also specify the order $d(s)$ of the zero of $f$ at each element $s$ of the set $S$ of isolated points of $E$. The present note improves this result by showing that each nonquasi-analytic class $C\left\{M_{n}\right\}$ contains such functions.
\end{abstract}

R. B. Hughes stated the foregoing interesting result in [1]. The purpose of this note is to present a short proof of Hughes' theorem based on properties of H. E. Bray's construction presented on pp. 79-84 of [2].

Suppose that $\left\{M_{n}\right\}_{n=1}^{\infty}$ is a sequence of positive numbers; set $\mu_{1}=M_{1}^{-1}$ and $\mu_{k}=\left(M_{k-1} / M_{k}\right), k>1$.

A $C^{\infty}$-function $\phi$ on $R$ belongs to the class $C\left\{M_{n}\right\}$ if there is a positive number $A_{\phi}$ such that $\|\phi\|_{\infty} \leqq A_{\phi}$ and $\left\|\phi^{(k)}\right\|_{\infty} \leqq A_{\phi}^{k} M_{k}, k \geqq 1$.

The theory of $C^{\infty}$-functions permits us to suppose, without loss of generality, that $\mu_{k} \geqq \mu_{k+1}, k \geqq 1$. Then the Denjoy-Carleman theorem [3, p. 376] tells $u$ s that the class $C\left\{M_{n}\right\}$ is a quasi-analytic class if, and only if, $\mu=\sum \mu_{k}<\infty$.

THEOREM. There is a function $f$ in $C\left\{M_{n}\right\}$ such that $f(x)=0 \Leftrightarrow x \in E$ and furthermore, for every $s \in S$, the order of the zero of $f$ at $s$ is $d(s)$.

Proof. We begin with some preliminaries. First, we apply Bray's construction to the characteristic function of the interval $[-\mu, \mu]$ to obtain a function $M$ in $C\left\{M_{n}\right\}$ with $A_{M}=1$ and $M(x)>0 \Leftrightarrow x \in(-2 \mu, 2 \mu)$. Next, we notice that it suffices to suppose that each component of the complement of $E$ has length $<1$. Then we observe that translating and stretching $M$ yields a function $g$ in $C\left\{M_{n}\right\}$ with $A_{g}=1 / 2, g(x)=0$ if $x \leqq 0$, and $g$ strictly increasing (even convex if you wish) on (say) [0,3]. For $a<b<a+1$, set $h(a, b, t)=g(t-a) g(b-t), t \in R$. Then [3, pp. 373-374] $h(a, b, t)>0 \Leftrightarrow t \in(a, b)$ and $A_{h(a, b, t)}=1$. Now we are ready to begin construction of the requisite function $f$. The first step is to let $\left\{\left(a_{i}, b_{i}\right)\right\}$ be an enumeration of the components of $R-E$ and set $h(t)=\sum_{i} p_{i} h\left(a_{i}, b_{i}, t\right)$,

Received by the editors October 16, 1972 and, in revised form, January 8, 1973.

AMS (MOS) subject classifications (1970). Primary 26A93, 54C50.

Key words and phrases. $C^{\infty}$-functions, non-quasi-analytic classes, zero sets. 
where the $p_{i}$ 's have absolute value one. Then $h(t)=0 \Leftrightarrow t \in E$. Moreover, integrating that $(k+2)$ nd derivative on $\left(a_{i}, b_{i}\right)$ permits us to assert that there are constants $P_{k}$ such that $\left|h^{(k)}(t)\right| \leqq P_{k} \mid\left(t-a_{i}\right)^{2}\left(b_{i}-t\right)^{2}, t \in\left(a_{i}, b_{i}\right)$, $k=1,2, \cdots$. Hence, $h^{(1)} \equiv 0$ on $E$; and if we suppose that $h^{(k)}(t)=0$ for all $t \in E$, then it follows that $h^{(k+1)} \equiv 0$ on $E$. Thus $h \in C\left\{M_{n}\right\}$ and $A_{h}=1$. If $S=\varnothing$, we are done; otherwise, the second step is to adjust the order of zero at the isolated points of $E$. To this end we recall that if $s \in S$, the two components, $\left(a_{i}, s\right)$ and $\left(s, b_{j}\right)$, of $R-E$ abut at $s$. Then we denote by $\left(\alpha_{s}, \beta_{s}\right)$ the segment of length $\frac{1}{2} \min \left\{s-a_{i}, b_{j}-s\right\}$ centered at $s$. Next we notice that since $n \mu_{n} \rightarrow 0$, there is a constant $Q_{s}>0$ such that the function, $g_{s}$, defined by $g_{s}(t)=Q_{s}(t-s)^{d(s)} h\left(\alpha_{s}, \beta_{s}, t\right)$ has order $d(s)$ at $s$, is zero on a neighborhood of $E-\{s\}$ and belongs to $C\left\{M_{n}\right\}$ with $A_{g_{s}}=1$. Set $y(t)=$ $\sum_{s} q_{s} g_{s}(t)$, where $\left|q_{s}\right|=1$. One final adjustment is necessary; it remains to specify the $p_{i}$ 's and $q_{s}$ 's so that $f=h+y$ is nonzero on $R-E$ as follows. Enumerate $S$. Set $q_{s_{1}}=1$. Specify $q$ at abuting points of $S$ so that $y$ is either nonnegative or nonpositive on each $\left(a_{i}, b_{i}\right)$. Iterate this procedure as far as possible. Either $q$ is defined on all of $S$ or there is a first $s_{j}$ not specified. Then set $q_{s_{j}}=1$ and repeat the process. In this way, all the $q_{s}^{\prime}$ 's are specified. Set $p_{i}=-1$ if $y$ is negative at some point of $\left(a_{i}, b_{i}\right)$ and $p_{i}=1$ otherwise.

\section{REFERENCES}

1. R. B. Hughes, Zero sets of functions from non-quasi-analytic classes, Proc. Amer. Math. Soc. 27 (1971), 539-542.

2. S. Mandelbrojt, Analytic functions and classes of infinitely differentiable functions, Rice Inst. Pamphlet 29, no. 1 (1942). MR 3, 292.

3. W. Rudin, Real and complex analysis, McGraw-Hill, New York, 1966. MR 35 \#1420.

Department of Mathematics, Colorado State University, Fort Collins, Colorado 80521 